\title{
Correlation between cardiorespiratory coupling and six-minute walking tests in patients with chronic obstructive pulmonary disease
}

\section{Yu-Chen Huang}

Chang Gung Memorial Hospital https://orcid.org/0000-0001-6682-4035

Ting-Yu Lin

Chang Gung Memorial Hospital

Hau-Tieng Wu

Duke University

Po-Jui Chang

Chang Gung Memorial Hospital

Chun-Yu Lo

Chang Gung Memorial Hospital

Tsai-Yu Wang

Chang Gung Memorial Hospital

Chih-Hsi Scott Kuo

Chang Gung Memorial Hospital

Shu-Min Lin

Chang Gung Memorial Hospital

Fu-Tsai Chung

Chang Gung Memorial Hospital

Horng-Chyuan Lin

Chang Gung Memorial Hospital

Meng-Heng Hsieh

Chang Gung Memorial Hospital

Yu-Lun Lo ( $\sim$ loyulun@hotmail.com )

Chang Gung Memorial Hospital

\section{Research}

Keywords: Heart-lung interaction, synchrogram index, six-minute walking distance, distance saturation product

Posted Date: March 20th, 2020 
DOI: https://doi.org/10.21203/rs.3.rs-18112/v1

License: (c) (i) This work is licensed under a Creative Commons Attribution 4.0 International License. Read Full License 


\section{Abstract}

Background : Cardiovascular disease is a common comorbidity and cause of mortality among patients with chronic obstructive pulmonary disease (COPD). However, the interaction between the heart and lungs in COPD patients has yet to be fully elucidated.

Aim : Our objective in this study was to characterize cardiorespiratory interactions in terms of cardiorespiratory coupling (CRC) using the synchrogram index of the heart rate and respiration flow signals.

Methods : This prospective study examined 10 normal subjects and 55 COPD patients. Linear regression and forward stepwise regression were used to determine the correlation between the synchrogram index and the six-minute walking test.

Results : K-means clustering analysis was used to separate the 55 COPD patients into a synchronized group (median 0.89 (0.64-0.97), n=43) and a desynchronized group (median $0.23(0.02-0.51), \mathrm{n}=12$ ) based on the synchrogram index. In this study, the synchrogram index was significantly correlated with the six-minute walking distance ( $r 2=0.3$, sigma $T=0.02)$ and the distance saturation product $(r 2=0.3$, sigma $T=0.03)$. Note that age was a significant confounding factor.

Conclusion : The synchrogram index shows clinical potential for the stratification of COPD patients for treatment.

\section{Introduction}

Chronic obstructive pulmonary disease (COPD) is characterized by irreversible airflow limitations resulting from the destruction of the alveolar attachment. [1] Possible causes include exposure or inhalation of noxious gases or particles and systemic inflammation. COPD has been shown to affect nutritional status, exercise tolerance, and the cardiovascular system. [2] The prevalence of cardiovascular disease as a comorbidity and cause of mortality underlines the need to consider the interaction between the heart and lungs in patients with COPD. [3-5]

Heart-lung interactions can be classified according to the underlying mechanisms: (i) respiratory sinus arrhythmia, (ii) cardioventilatory coupling, and (iii) respiratory stroke volume synchronization. [6] During inspiration, central inspiratory drive [7] and negative intrathoracic pressure [8] both contribute to an increase in heart rate. [9] Negative intrathoracic pressure promotes filling of the right ventricle and impedes filling of the left ventricle. [10] A decrease in arterial blood pressure tends to increase respiratory rate and tidal volume through the baroreflex. [11]

Several studies have described heart rate variability (HRV) and its relationship with the respiratory system among subjects with COPD. Researchers have found that cardiac autonomic dysfunction is more prevalent among COPD patients than among normal subjects, due presumably to inspiratory muscle 
weakness [12,13] and poor health-related quality of life. [14] Note however that heart-lung interactions in COPD subjects have not been fully elucidated. Synchrogram is a widely applied signal processing tool that facilitates the detection of coupling between two oscillatory signals. $[15,16]$ It has been used to study the heart-lung interactions in normal subjects[17], infants [18], and schizophrenia patients. [19] In addition, it is applied to identify sleep stages [20] in patients with obstructive sleep apnea. Essentially, this analysis can be divided into two steps: (1) detecting the instantaneous phase; and (2) quantifying the degree of phase coupling. The term instantaneous phase describes the oscillatory status of a waveform signal at a given point in time. The degree of coupling is calculated by obtaining the synchrogram of the instantaneous phases of two oscillatory signals. In this prospective study, we explored the phase coupling of the instantaneous heart rate and respiratory flow signal with the aim of elucidating an important mechanism underlying heart-lung interactions (i.e., respiratory sinus arrhythmia) in patients with COPD. [20]

\section{Methods}

\section{Study Design and Patients}

This prospective study included normal subjects and patients with a clinical diagnosis COPD, based on the Global Initiative for Obstructive Lung Disease Criteria (GOLD). The study was conducted at Chang Gung Memorial Hospital during January and December in 2019. Patients with heart failure (ejection fraction $<40 \%$ ), known malignancy, or atrial fibrillation as well as those using anti-arrhythmic agents for arrhythmia or oxygen were excluded. All COPD patients underwent cardiac echo analysis, biochemical analysis (eosinophils, high sensitivity C-reactive protein (HS-CRP), and IgE), pulmonary function tests, a six-minute walking test, and a coupling test during the first visit. Clinical profiles, a list of inhalation medicines, and acute exacerbation history [21] were also recorded (Figure 1). A total of 55 patients with COPD (69 (51-84) years old, 54 male) and ten normal subjects (64.5 (50-71) years old, 5 male) were enrolled. All participants signed informed consent prior to enrollment. The study was approved by the Ethics Committee of Chang Gung Memorial Hospital, Linkou, Taiwan (201702150B0).

\section{Phase synchronization analysis}

\section{Instrumentation}

Experiments were performed in a quiet room with the temperature maintained at $22-24^{\circ} \mathrm{C}$. Participants were instructed to avoid bronchodilators, such as beta-2 agonists, xanthene derivatives, alcohol, and coffee prior to the test. The chest skin was abraded using gel and then cleaned using alcohol to reduce electrode impedance prior to the attachment of electrocardiogram (ECG) electrodes. Prior to the examination, recordings were obtained of blood pressure, heart rate, and oxygen saturation. The subjects wore a pulse oximeter on the index finger and ECG electrodes on the chest wall. To avoid signal quality issues, the patient practiced breathing through the flow tube for 1 min before recording. ECG signals and flow signals were then recorded continuously for 5 min using three Actiwave devices (CamNtech Ltd, 
Cambridge, UK). The recorded signals were transferred in European Data Format to LabChart 8 software (ADInstruments, Dunedin, New Zealand), and then exported to text files for analysis.

\section{Signal Processing and Synchrogram index}

$\mathrm{R}$ peaks were detected using a standard $\mathrm{R}$ peak detection algorithm, and the time differences between consecutive $R$ peaks were converted into an instantaneous heart rate (IHR) time series using a standard interpolation algorithm. [22] Instantaneous phases of the respiratory signals and IHR time series were extracted using the synchrosqueezing transform (SST). [23] The time-varying broad pass issue associated with the Hilbert transform was avoided using the generalized SST form. After obtaining the instantaneous phases of the IHR (denoted as $\Phi_{H}$ ) and the respiratory signal (denoted as $\Phi_{R}$ ), the synchrogram was used to quantify phase synchronization. $[17,24]$ Note that a synchrogram is a graphical tool used to depict phase coupling between two oscillatory signals. In the current study, we first obtained the timestamps $t_{k}$ at the point where the IHR phase attained 0 (i.e., $\Phi_{H}\left(t_{k}\right) \bmod 2 \pi=0$ ). We then measured the respiratory phase at $t_{k}$ as follows: $\psi\left(t_{k}\right)=1 / 2 \pi\left[\Phi_{R}\left(t_{k}\right) \bmod 2 \pi\right]$.

Plotting $\psi\left(t_{k}\right)$ against $t_{k}$ made it possible to obtain a horizontal stripe in cases where the IHR and respiratory signals were synchronized; otherwise, we obtained scattered points. Cardiorespiratory synchronization was quantified by measuring the synchrogram index $\lambda$ [24] as follows:

$\lambda=\frac{1}{M}\left[\left(\sum_{t_{k}=1}^{M} \sin 2 \pi \psi\left(t_{k}\right)\right)^{2}+\left(\sum_{t_{k}=1}^{M} \cos 2 \pi \psi\left(t_{k}\right)\right)^{2}\right]$,

where $\mathrm{M}$ is the number of detected cycles in the IHR. The synchronization index was closer to 1 in synchronized systems and closer to 0 in unsynchronized systems.

\section{Statistical Analysis}

All results are presented as median (range) or mean \pm standard deviation. The nonparametric exact twotailed Mann-Whitney $U$ test was used to determine the statistical significance between two groups of continuous variables, and Fisher's exact tests were used for categorical variables. Variance differences were evaluated using the F-test. Pearson's correlation was used to examine the association between distance, distance saturation product, and synchrogram index. Forward stepwise multiple regression analysis of the distance and distance saturation product was used to evaluate the contribution of age, body mass index, and synchrogram index. All reported $P$ values were two-sided, with $P<0.05$ considered statically significant. All data were analyzed using $R$ version 3.5.2 ( $R$ foundation for statistical computing).

\section{Results}

\section{Baseline normal subjects and patient characteristics}

The normal group included a larger proportion of females (5 (50\%)) and non-smokers (9 (90\%)) (Table 1). Among the 55 patients in the experiment group, 54 (98.2\%) were male, 49 (89.1\%) were smokers, 36 (66.7\%) were in allergic status, and only $1(1.9 \%)$ patient fulfilled the criteria of Asthma-COPD overlap 
(ACO). [25, 26] The median body mass index (BMI) was 24.7 (range 16.7-32.1), modified medical research council (mMRC) was 1 (range 0-4), and COPD assessment test (CAT) was 10 (range 2-29). The median ejection fraction was 65.5 (range 52-90), the eosinophil count was 129 (range 0-615.6), and the IgE level was 59.7 (2-1652). In addition, 4 (7.3\%) patients were using long-acting $\beta_{2}$ agonist (LABA) alone, 1 (1.8\%) was using long-acting muscarinic antagonist (LAMA) alone, 20 (36.4\%) patients were using combination therapy of LABA with LAMA, 3 (5.5\%) were using a combination therapy of LABA with inhaled corticosteroids (ICS), 26 (47.3\%) patients were using triple therapy of LABA with LAMA and ICS, and 5 (9.1\%) patients were taking oral corticosteroids with inhalation therapy. Finally, 19 (34.5\%) patients had a history of acute exacerbation one year prior to enrollment in the study (Table 2).

\section{ECG, flow signal, CRC data, and synchrogram index}

Figures $2 \mathrm{a}$ and $2 \mathrm{~b}$ illustrate CRC analysis based on the synchrogram of IHR and respiratory flow signals. The synchrogram index values were as follows: normal group $(0.86 ; 0.73-0.95)$ and COPD patients $(0.87$; 0.02-0.97), as listed in Table 1. There are no definitions of good or poor synchronization; therefore, we applied K-means cluster analysis (a well-known unsupervised learning algorithm) [27] to the synchrogram index in order to identify a synchronized group $(n=43)$ and desynchronized group $(n=12)$ (Figure 3a, 3b). The median synchrogram index values were as follows: synchronized group $(0.89 ; 0.64-0.97)$ and desynchronized group $(0.23 ; 0.02-0.51)$, as listed in Table 2 . Overall, subjects in the synchronized group were younger (69 (51-84) vs 77 (52-84), p= 0.03) and had a lower BMI (24.2 (16.7-32) vs 26.2 (20.3-30.8), $p=0.04)$. No significant between-group differences were observed in terms of gender, smoking status, allergic status, therapies, or history of acute exacerbation (Table 2).

\section{Comparing coupling tests with six-minute walking test}

Patients in the synchronized group presented longer walking distances (468 (328-624) vs. 408 (182-517), unit $=m, p=0.009)$ and a higher distance saturation product (421.2 (255.6-536.6), vs $373.2(149.2-464.6)$, unit $=m \%, p=0.02)$ (Table 3$)$. The synchrogram index correlated significantly with distance $(r=0.42, p$ $=0.0012)$ (Figure $3 c$ ) and distance saturation product $(r=0.4, p=0.002)$ (Figure 3d). Also note that even after discounting the effects of age and BMI, the synchrogram index remained a significant factor correlating with distance and distance saturation product. (Supplement 1, 2)

\section{Discussion}

CRC has been widely studied in normal subjects [17] and infants [28], and as an indication of sleep stages; $[20,29]$ however, it has not been examined in the context of COPD. In this study, we compared COPD patients and normal subjects using the results of synchronization analysis. We analyzed the interaction between IHR and respiratory signals. We also used the synchrogram index to quantify the interaction between the heart and lungs. The synchrogram index distribution was wide among COPD patients and narrow among normal subjects. Using k-means clustering to classify COPD patients as 
synchronized or desynchronized, we found that the synchrogram index distribution in the synchronized group was similar to that among normal subjects.

This study led to two major findings. The distance and distance saturation product from six-minute walking tests were significantly lower in the desynchronized group than in the synchronized group. Even after discounting the effects of age and BMI in a forward stepwise regression model, the synchrogram index remained a significant factor correlating with distance and distance saturation product.

Researchers have previously demonstrated that six-minute walking distance is an important predictor of survival in COPD patients [30,31] and heart failure patients. [32] Our study revealed that walking distance was lower among desynchronized patients; however, these results could not be confirmed from the perspective of outcomes due to inadequate follow-up duration. The poor walking distance demonstrated by COPD patients can be attributed to desaturation, the severity of emphysema, dyspnea scores, and inspiratory capacity as determined by ECLIPSE (Evaluation of COPD longitudinally to identify predictive surrogate endpoints). [33] Note that poor six-minute walking distance was also observed in patients presenting with heart failure with preserved ejection function. [34] Del Buono et al. reported that several comorbid conditions, such as skeletal muscle dysfunction, impaired autonomic regulation, and nutritional factors, may coexist and contribute to exercise intolerance in patients suffering from heart failure. [35] It has also been reported that impaired aerobic function contributes to low exercise intolerance in patients with COPD and in those suffering heart failure, due to negative cardiopulmonary-muscular interaction. [36] Desaturation levels, Borg score, inspiratory capacity, flow limitations ( $\mathrm{FEV}_{1}, \%$ predicted), and dynamic hyperinflation ( $\Delta$ inspiratory capacity) were similar in these two groups, and these showed no connection to walking distance in this study. This is an indication that walking distance is independent of desaturation and impaired pulmonary or heart function. Instead, it appears that nutritional status, peripheral muscle condition, [37] oxygen utilization by peripheral muscle, and negative cardiopulmonarymuscle interactions [36] should be taken into account.

Distance saturation product (i.e., the product of nadir saturation during exercise and walking) is a known predictor of mortality among patients with bronchiectasis [38] and interstitial lung disease. [39] Nonetheless, it has not yet been discussed in the context of COPD. In this study, patients in the desynchronized group presented a lower distance saturation product, which suggests an elevated likelihood of poor outcomes. Our results indicate that nadir saturation was not correlated with the synchrogram index, suggesting that distance contributed to distance saturation product but not desaturation. A strong heart-lung interaction may improve ventilation and perfusion matching, resulting in better oxygen transport; [40] however, we did not observe any discrepancy between the synchronized and desynchronized groups in terms of saturation. This may be explained by the fact that we excluded patients who were using oxygen on a daily basis. Note that there may be a link between desaturation and coupling in those patients. To further study this relationship, we should explore COPD patients who used oxygen daily. There is also the possibility that the patients stopped walking prior to the onset of desaturation. To examine this possibility, we need more data and carry out the subgroup analysis in patients who have poor performance in walking distance to evaluate the cause of stopping walking. 
This study faced a number of limitations. First, despite measuring and quantifying the coupling between respiration flow signals and ECG signals, we found no indications of causality. Second, the strict inclusion criteria prevented us from analyzing patients using oxygen on a daily basis. Third, most of the patients in the study were male and all were of East Asian decent; i.e., this sample is not representative of COPD patients overall. Finally, the follow-up time of this preliminary study was of insufficient duration to evaluate mortality outcomes and cardiac vascular events. We will continue monitoring the subjects in this study in order to observe the clinical impact of synchronization in heart-lung interactions.

\section{Conclusions}

This study conducted CRC analysis to describe respiratory sinus arrhythmia, a mechanism underlying heart-lung interactions in COPD patients. CRC is significantly correlated with six-minute walking distance and distance saturation product. Note that age was a significant confounding factor. The synchrogram index demonstrates clinical potential for the stratification of COPD patients for treatment.

\section{Abbreviations}

ACO: Asthma-COPD overlap; AE: acute exacerbation; BMI: body mass index; CAT: chronic obstructive pulmonary disease assessment test; COPD: chronic obstructive pulmonary disease; $\mathrm{CRC}$ :

cardiorespiratory coupling; ECG: electrocardiogram; $\mathrm{ETCO}_{2}$ sensor: end tidal $\mathrm{CO}_{2}$ sensor; $\mathrm{FEV}_{1}$ : forced expiratory volume in $1^{\text {st }}$ second; FVC: forced vital capacity; GOLD: Global Initiative for Obstructive Lung Disease Criteria; HRV: heart rate variability; HS-CRP: high sensitivity C-reactive protein; ICS: inhaled corticosteroids; IHR: instantaneous heart rate; mMRC: modified medical research council; LABA: longacting beta agonists; LAMA: long-acting antimuscarinic agents; OCS: oral corticosteroids; SST: synchrosqueezing transform

\section{Declarations}

Funding

This study was supported by a grant from the Taiwan Ministry of Science and Technology, 107-2314-B182A-145-MY2.

\section{Author Information}

Yu-Chen Huang is the first author

\section{Affiliations}

${ }^{1}$ Department of Thoracic Medicine and Critical care, Saint Paul's Hospital, Taoyuan, Taiwan

${ }^{2}$ Department of Thoracic Medicine, Chang Gung Memorial Hospital, Taipei, Taiwan 
${ }^{3}$ College of Medicine, Chang Gung University, Taoyuan, Taiwan

${ }^{4}$ Department of Mathematics, Duke University, Durham, NC, USA

${ }^{5}$ Department of Statistical Sciences, Duke University, Durham, NC, USA

\section{Contributions}

TY, HT, PJ, CY and YL conceived and designed the analysis. YC designed and performed the statistical analyses. All authors were involved in data analysis, data interpretation, and preparation of the final manuscript. The authors read and approved the final manuscript.

\section{Corresponding author}

Correspondence to Yu-Lun Lo

\section{Ethics declarations}

\section{Ethics approval and consent to participate}

The study was conducted and approved by local institutional review boards (details previously published). Patients provided signed informed consent prior to screening.

\section{Consent for publication}

Not applicable.

\section{Competing interests}

All authors all declare that they have no competing interests.

\section{Acknowledgements}

This study was supported by a grant from the Taiwan Ministry of Science and

Technology, 107-2314-B-182A-145-MY2.

\section{References}

1. Scichilone N, Bruno A, Marchese R, Vignola AM, Togias A, Bellia V. Association between reduced bronchodilatory effect of deep inspiration and loss of alveolar attachments. Respir Res 2005; 6: 55.

2. Rabe KF, Hurd S, Anzueto A, Barnes PJ, Buist SA, Calverley P, Fukuchi Y, Jenkins C, Rodriguez-Roisin $\mathrm{R}$, Van Weel C. Global strategy for the diagnosis, management, and prevention of chronic obstructive 
pulmonary disease: GOLD executive summary. Am J Respir Critical Care Med 2007; 176: 532-5.

3. Fabbri L, Luppi F, Beghé B, Rabe KF. Complex chronic comorbidities of COPD. Eur Respir J 2008; 31 : 204-12.

4. Godtfredsen NS, Lam TH, Hansel TT, Leon ME, Gray N, Dresler C, Burns DM, Prescott E, Vestbo J. COPD-related morbidity and mortality after smoking cessation: status of the evidence. Eur Respir $\mathrm{J}$ 2008; 32: 844-53.

5. Nussbaumer-Ochsner Y, Rabe KF. Systemic manifestations of COPD. Chest 2011;139:165-73.

6. Elstad M, O'Callaghan EL, Smith AJ, Ben-Tal A, Ramchandra R. Cardiorespiratory interactions in humans and animals: rhythms for life. Am J Physiol-Heart C 2018; 315: 6-17.

7. Baekey DM, Dick TE, Paton JF. Pontomedullary transection attenuates central respiratory modulation of sympathetic discharge, heart rate and the baroreceptor reflex in the in situ rat preparation. Exp Physiol 2008; 93: 803-16.

8. Lopes O, Palmer J. Proposed respiratory 'gating'mechanism for cardiac slowing. Nature 1976; 264: 454.

9. Ben-Tal A, Shamailov SS, Paton JF. Central regulation of heart rate and the appearance of respiratory sinus arrhythmia: New insights from mathematical modeling. Math Biosci 2014; 255: 71-82.

10. Elstad, M. Respiratory variations in pulmonary and systemic blood flow in healthy humans. Acta Physiol 2012; 205: 341-48.

11. De Burgh Daly, M. Interactions between respiration and circulation. Compr Physiol 2011: 529-94.

12. Reis MS, Arena R, Deus AP, Simões RP, Catai AM, Borghi-Silva A. Deep breathing heart rate variability is associated with respiratory muscle weakness in patients with chronic obstructive pulmonary disease. Clinics 2010; 65: 369-75.

13. Goulart CDL, Simon JC, Schneiders PDB, San Martin EA, Cabiddu R, Borghi-Silva A, Trimer R, da Silva ALG. Respiratory muscle strength effect on linear and nonlinear heart rate variability parameters in COPD patients. Int J Chron Obstruct Pulmon Dis 2016; 11: 1671.

14. Van Gestel AJ, Kohler M, Steier J, Teschler S, Russi EW, Teschler H. Cardiac autonomic dysfunction and health-related quality of life in patients with chronic obstructive pulmonary disease. Respirology 2011; 16: 939-46.

15. Rosenblum MG, Pikovsky AS, Kurths J. Phase synchronization of chaotic oscillators. Phys Rev Lett 1996; 76: 1804.

16. Pikovsky AS, Rosenblum MG, Osipov GV, Kurths J. Phase synchronization of chaotic oscillators by external driving. Physica D1997; 104: 219-38.

17. Schäfer C, Rosenblum MG, Kurths J, Abel HH. Heartbeat synchronized with ventilation. Nature 1998; 392: 239.

18. Mrowka R, Patzak A, Rosenblum M. Quantitative analysis of cardiorespiratory synchronization in infants. Int J Bifurcat Chaos 2000; 10: 2479-88. 
19. Schulz S, Bär KJ, Voss A. Analyses of heart rate, respiration and cardiorespiratory coupling in patients with schizophrenia. Entropy 2015; 17: 483-501.

20. Niizeki K, Saitoh T. Association between phase coupling of respiratory sinus arrhythmia and slow wave brain activity during sleep. Front Physiology 2018; 9:1338.

21. Rodriguez-Roisin R. Toward a Consensus Definition for COPD Exacerbations. Chest 2000; 117: 398401.

22. Camm AJ, Malik M, Bigger JT, Breithardt G, Cerutti S, Cohen R, Coumel P, Fallen EL, Kennedy HL, Kleiger RE. Heart rate variability: standards of measurement, physiological interpretation and clinical use. Task Force of the European Society of Cardiology and the North American Society of Pacing and Electrophysiology. Eur Heart J 1996; 17:354-81.

23. Daubechies I, Lu J, Wu HT. Synchrosqueezed wavelet transforms: An empirical mode decompositionlike tool. Appl Comput Harmon A 2011; 30: 243-61.

24. Nguyen CD, Wilson SJ, Crozier S. Automated quantification of the synchrogram by recurrence plot analysis. IEEE Trans Biomed Eng 2012; 59: 946-55.

25. Sin DD, Miravitlles M, Mannino DM, Soriano JB, Price D, Celli BR, Leung JM, Nakano Y, Park, HY, Wark PA. What is asthma- COPD overlap syndrome? Towards a consensus definition from a round table discussion. Eur Respir J 2016; 48: 664-73.

26. Maselli DJ, Hardin M, Christenson SA, Hanania NA, Hersh CP, Adams SG, Anzueto A, Peters JI, Han MK, Martinez FJ. Clinical approach to the therapy of asthma-COPD overlap. Chest 2019; 155: 168-77.

27. Hartigan J, Wong M. A K-Means Clustering Algorithm. J R Stat Soc C-APPL 1979; 28: 100-8.

28. Rosenblum MG, Cimponeriu L, Bezerianos A, Patzak A, Mrowka R. Identification of coupling direction: application to cardiorespiratory interaction. Phys Rev E 2002; 65: 041909.

29. Bartsch R, Kantelhardt JW, Penzel T, Havlin S. Experimental evidence for phase synchronization transitions in the human cardiorespiratory system. Phys Rev Lett 2007; 98: 054102.

30. Polkey MI, Spruit MA, Edwards LD, Watkins ML, Pinto-Plata V, Vestbo J, Calverley PM, Tal-Singer R, Agustí A, Bakke PS. Six-minute-walk test in chronic obstructive pulmonary disease: minimal clinically important difference for death or hospitalization. Am J Respir Crit Care Med 2013; 187: 382-6.

31. Pinto-Plata V, Cote C, Cabral H, Taylor J, Celli B. The 6-min walk distance: change over time and value as a predictor of survival in severe COPD. Eur Respir J 2004; 23: 28-33.

32. Rubim VSM, Drumond Neto C, Romeo JLM, Montera MW. Prognostic value of the six-minute walk test in heart failure. Arq Bras Cardio/ 2006; 86: 120-5.

33. Spruit MA, Watkins ML, Edwards LD, Vestbo J, Calverley PMA, Pinto-Plata V, Celli BR, Tal-Singer R, Wouters EF. Determinants of poor 6-min walking distance in patients with COPD: the ECLIPSE cohort. Respir Med 2010; 104: 849-57.

34. Boyes N, Eckstein J, Pylypchuk S, Lahti D, Butcher SJ, Marciniuk DD, Dewa DM, Wells CR, Haykowsky MJ, Tomczak CR. Peak Exercise and Post-Exercise Recovery Oxygen Uptake and Muscle Oxygenation 
in Patients with Heart Failure and Preserved Ejection Fraction and Healthy Matched Adults. FASEB $J$ 2019; 33: 461.

35. Del Buono MG, Arena R, Borlaug BA, Carbone S, Canada JM, Kirkman DL, Garten R, RodriguezMiguelez P, Guazzi M, Lavie CJ. Exercise intolerance in patients with heart failure: JACC State-of-theArt Review. J Am Coll Cardiol 2019; 73: 2209-25.

36. Rocha A, Arbex FF, Sperandio PA, Mancuso F, Marillier M, Bernard AC, Alencar MCN, O'Donnell DE, Neder JA. Exercise intolerance in comorbid COPD and heart failure: the role of impaired aerobic function. Eur Respir J 2019; 53: 1802386.

37. McDonald CM, Henricson EK, Han JJ, Abresch RT, Nicorici A, Elfring GL, Atkinson L, Reha A, Hirawat S, Miller LL. The 6-minute walk test as a new outcome measure in Duchenne muscular dystrophy. Muscle Nerve 2010; 41: 500-10.

38. Hsieh MH, Fang YF, Chung FT, Lee CS, Chang YC, Liu YZ, Wu CH, Lin HC. Distance-saturation product of the 6-minute walk test predicts mortality of patients with non-cystic fibrosis bronchiectasis. Journal of thoracic disease 2017; 9: 3168.

39. Lettieri CJ, Nathan SD, Browning RF, Barnett SD, Ahmad S, Shorr AF. The distance-saturation product predicts mortality in idiopathic pulmonary fibrosis. Respir Med 2006; 100: 1734-41.

40. Yasuma F, Hayano JI. Respiratory sinus arrhythmia: why does the heartbeat synchronize with respiratory rhythm? Chest 2004; 125: 683-90.

\section{Tables}

Table 1 Clinical characteristics of total patients and normal subjects

\begin{tabular}{lllll}
\hline & Patients $(\mathrm{n}=55)$ & Normal subjects $(\mathrm{n}=10)$ & F-value & $\mathrm{p}$-value \\
\hline Age & $69(51-84)$ & $64.5(40-71)$ & 1.12 & 0.73 \\
\hline Gender, male & $54(98.2)$ & $5(50)$ & 15.50 & $<0.0001$ \\
\hline Smoker & $49(89.1)$ & $1(10)$ & 1.01 & 0.88 \\
\hline BMI & $24.7(16.7-32.0)$ & $23.5(20.3-29.8)$ & 0.91 & 0.96 \\
\hline Synchrogram Index & $0.87(0.02-0.97)$ & $0.86(0.73-0.95)$ & 4.62 & 0.03 \\
\hline
\end{tabular}

$\mathrm{P}$ values were calculated with analysis of variance. Abbreviation: BMI (Body mass Index) 
Table 2 Clinical characteristics of total patients, synchronized and desynchronized group

\begin{tabular}{|c|c|c|c|c|}
\hline & $\begin{array}{l}\text { Total } \\
(n=55)\end{array}$ & Synchronized (n=43) & Desynchronized (n=12) & p-value \\
\hline Age & $69(51-84)$ & $69(51-84)$ & $77(52-84)$ & 0.03 \\
\hline Gender, male & $54(98.2)$ & $42(97.7)$ & $12(100)$ & 0.59 \\
\hline Smoker & $49(89.1)$ & $38(88.4)$ & $11(91.7)$ & 0.75 \\
\hline BMI & $24.7(16.7-32.1)$ & $24.2(16.7-32.0)$ & $26.2(20.3-30.8)$ & 0.04 \\
\hline EF & $65.5(52-90)$ & $66.5(52-90)$ & $64.5(52-78)$ & 0.37 \\
\hline Eosinophil Count & $129(0-615.6)$ & $134.5(0-615.6)$ & $115.0(0-329.8)$ & 0.59 \\
\hline Allergy & $36(66.7)$ & $28(66.7)$ & $8(66.7)$ & 1.00 \\
\hline a-IgE & 59.7 (2-1652) & $65.2(3.59-1652)$ & $47.0(2-691)$ & 0.69 \\
\hline HS-CRP & $1.7(0.2-189.7)$ & $1.64(0.2-37.9)$ & $1.75(0.2-189.7)$ & 0.54 \\
\hline CAT & $10(2-29)$ & $10(2-29)$ & $6(3-29)$ & 0.62 \\
\hline mMRC & $1(0-4)$ & $1(0-3)$ & $1(0-4)$ & 0.84 \\
\hline Emphysema & $22(40)$ & $20(46.5)$ & $2(16.7)$ & 0.05 \\
\hline AE history & $19(34.5 \%)$ & $16(37.2 \%)$ & $3(25 \%)$ & 0.74 \\
\hline $\mathrm{ACO}$ & $1(1.8 \%)$ & $0(0 \%)$ & $1(8.3 \%)$ & 0.36 \\
\hline Synchrogram Index & $0.86(0.02-0.97)$ & $0.89(0.64-0.97)$ & $0.23(0.02-0.51)$ & $<0.0001$ \\
\hline \multicolumn{5}{|l|}{ Drugs } \\
\hline LABA & $4(7.3 \%)$ & $3(7.0 \%)$ & $1(8.3 \%)$ & 0.87 \\
\hline LAMA & $1(1.8 \%)$ & $1(2.3 \%)$ & $0(0)$ & 0.59 \\
\hline LABA+LAMA & $20(36.4 \%)$ & $15(34.9 \%)$ & $5(41.7 \%)$ & 0.87 \\
\hline LABA+ICS & $3(5.5 \%)$ & $2(4.7 \%)$ & $1(8.3 \%)$ & 0.62 \\
\hline Triple & $26(47.3 \%)$ & $22(51.2 \%)$ & 4 (33.3\%) & 0.27 \\
\hline OCS & $5(9.1 \%)$ & $4(9.3 \%)$ & $1(8.3 \%)$ & 0.93 \\
\hline
\end{tabular}

Significantly different from patients with synchronized and desynchronized $(P<0.05)$.Abbreviation: BMI (Body mass index), HS-CRP (High sensitivity C reactive protein), CAT(Chronic obstructive pulmonary disease assessment test), mMRC(modified medical research council), LABA (Long acting beta agonists), LAMA(Long acting antimuscarinic agents), ICS(inhaled corticosteroids), OCS(oral corticosteroids), AE(acute exacerbation) 
Table 3 Clinical characteristics of total patients, synchronized and desynchronized group

\begin{tabular}{|c|c|c|c|c|}
\hline & $\begin{array}{l}\text { Total } \\
(n=55)\end{array}$ & $\begin{array}{l}\text { Synchronized } \\
(\mathrm{n}=43)\end{array}$ & Desynchronized $(n=12)$ & p-value \\
\hline \multicolumn{5}{|c|}{ 6MWT median (range) } \\
\hline PreFVC(L) & $2.6(1.2-4.4)$ & $2.7(1.2-4.4)$ & $2.45(1.3-3.3)$ & 0.45 \\
\hline PreFVC (\%) & 78.7 (38-129) & 82.5 (39-129) & 77 (38-96) & 0.97 \\
\hline PreFEV1(L) & $1.5(0.5-2.7)$ & $1.39(0.6-2.7)$ & $1.43(0.5-2.0)$ & 0.65 \\
\hline PreFEV1(\%) & $56.7(18-102)$ & $55.5(18-102)$ & $62.5(18-76)$ & 0.85 \\
\hline PreFEV1/FVC & $0.56(0.31-0.72)$ & $0.57(0.31-0.72)$ & $0.56(0.35-0.7)$ & 0.89 \\
\hline PostFVC(L) & $2.7(1.1-4.4)$ & $2.7(1.1-4.4)$ & $2.7(1.4-3.3)$ & 0.45 \\
\hline PostFVC \% & $81(42-130)$ & $81.5(42-130)$ & $87(43-96)$ & 0.87 \\
\hline PostFEV1 (L) & $1.47(0.53-2.82)$ & $1.44(0.67-2.82)$ & $1.49(0.53-2.09)$ & 0.67 \\
\hline PostFEV1(\%) & $60(21-105)$ & $57(22-105)$ & $60(21-79)$ & 0.75 \\
\hline PostFEV1/FVC & $0.59(0.35-0.73)$ & $0.59(0.35-0.73)$ & $0.56(0.37-0.70)$ & 0.92 \\
\hline Pre-HR & $83(57-109)$ & 83.5 (57-109) & $80.5(60-98)$ & 0.50 \\
\hline Post-HR & 107 (69-149) & $108(69-149)$ & $105(70-122)$ & 0.44 \\
\hline Pre-Brog & $0(0-3)$ & $0(0-3)$ & $1(0-3)$ & 0.04 \\
\hline Post-Brog & $4(1-7)$ & $4(2-7)$ & $4(1-7)$ & 0.42 \\
\hline pre_spO2 & 95 (88-99) & 95.5 (88-99) & 95 (90-98) & 0.15 \\
\hline post_spO2 & $90.5(75-96)$ & $90.5(75-96)$ & $90(80-95)$ & 0.72 \\
\hline pre_IC & 1.78 (0.92-2.77) & 1.78 (1.07-2.77) & $1.73(0.92-2.04)$ & 0.16 \\
\hline post_IC & $1.73(0.94-2.83)$ & $1.73(0.97-2.83)$ & $1.7(0.94-2.43)$ & 0.41 \\
\hline$\Delta \mathrm{IC}$ & $0(-1.1-0.55)$ & $-0.02(-0.49-0.55)$ & $0.11(-1.1-0.28)$ & 0.37 \\
\hline$\Delta \mathrm{spO} 2$ & $-4.5(-22 \sim 0)$ & $-4.5(-22 \sim 0)$ & $-4.0(-18 \sim 0)$ & 0.97 \\
\hline Distance & $456(182-624)$ & $468(328-624)$ & $408(182-517)$ & 0.009 \\
\hline DSP & $411.1(149.2-536.6)$ & $421.2(255.6-536.6)$ & $373.2(149.2-464.6)$ & 0.02 \\
\hline
\end{tabular}

*Significantly different from patients with Synchronized and Desynchronized $(\mathrm{P}<0.05)$.Abbreviation: $6 \mathrm{MWT}$ (six minutes walking test), Pre(preexercise), Post(post-exercise), FVC (Forced vital capacity), FEV1 (Forced expiratory volume in 1st second), HR (Heart rate), $\Delta \mathrm{IC}$ (change of inspiratory capacity), $\triangle$ spO2 (change of oxyhemoglobin saturation by pulse oximetry), DSP (Distance saturation product)

Figures 


\begin{tabular}{|c|c|}
\hline Screening & Patients with \\
chronic obstructive lung disease \\
during $2019 / 01-2020 / 01$ \\
$\mathrm{~N}=60$
\end{tabular}

\section{Enrollment}

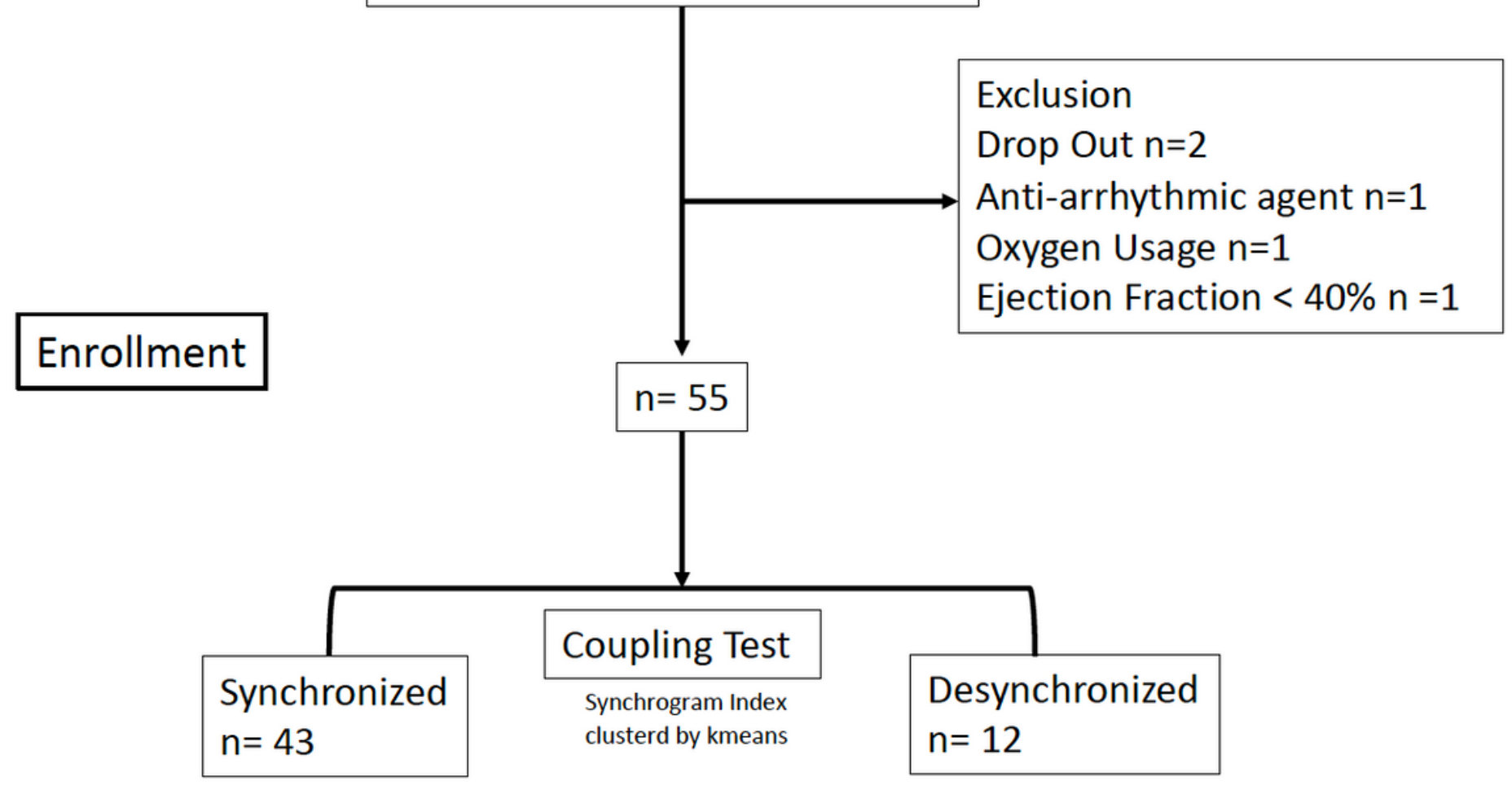

\section{Figure 1}

Flow chart. 
(a) a1

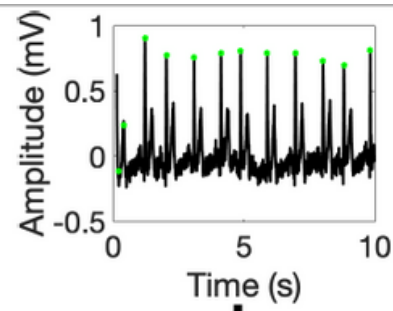

a2

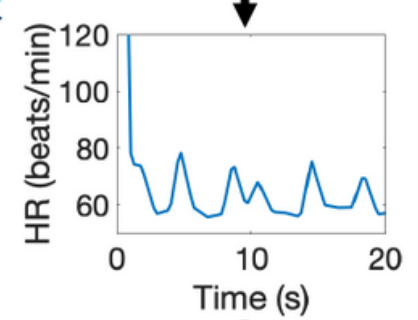

a3

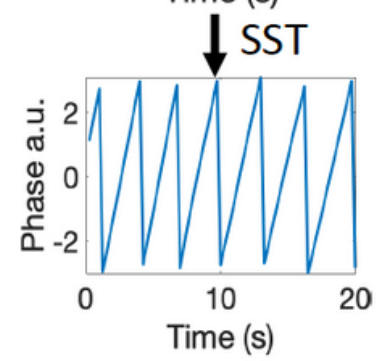

a4
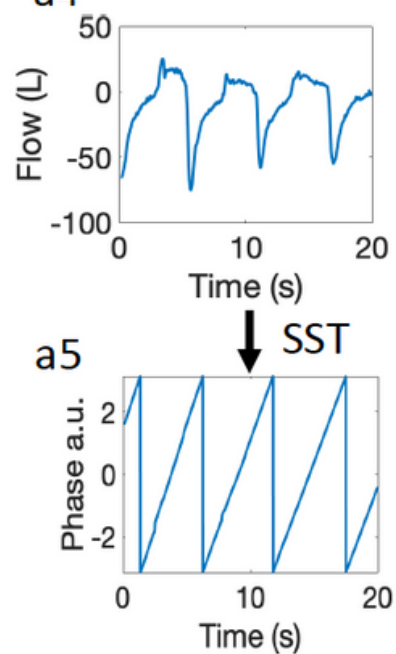

a6

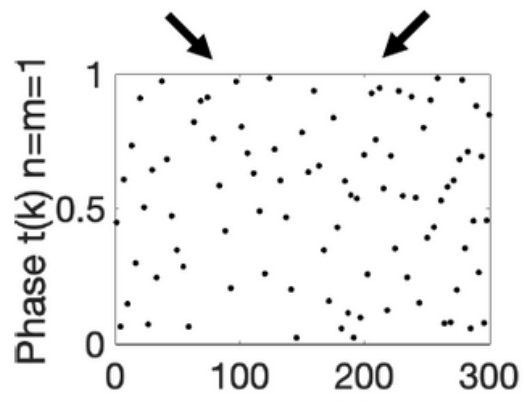

Synchrogram Index $=\mathbf{0 . 3 5}$ (b) b1

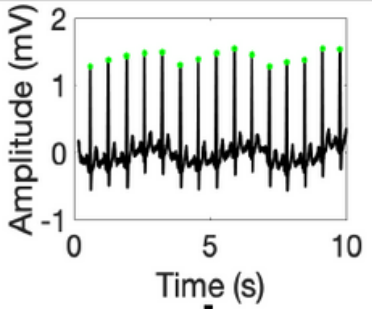

b2

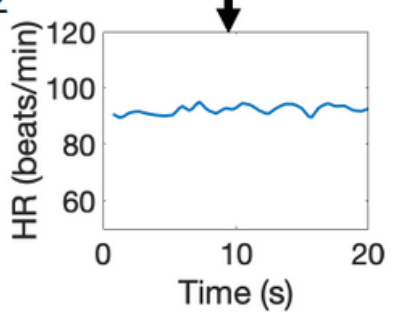

b4

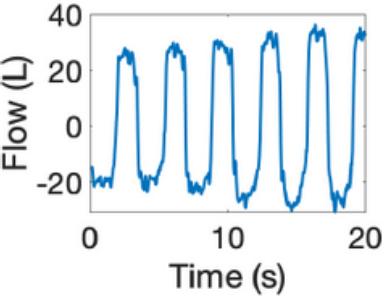

b3
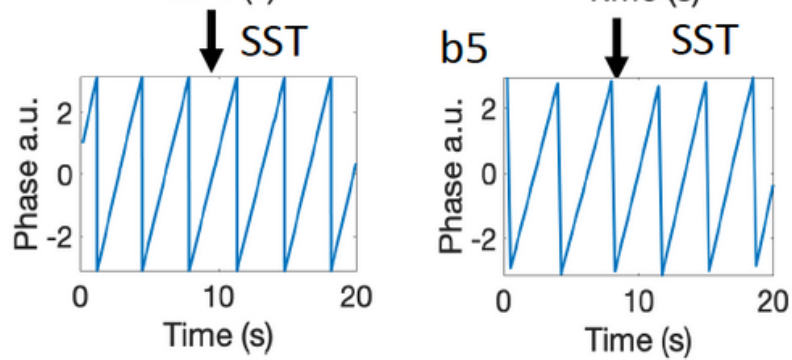

b6

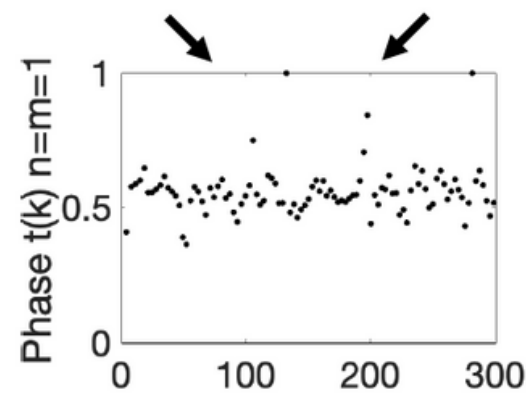

Synchrogram Index $=0.89 \quad$ Time (s)

Figure 2

(a1) Electrocardiogram, (a2) instantaneous heart rate (IHR), (a3) phase of the IHR extracted by the synchrosqueezing transform (SST), (a4) respiratory flow signal, (a5) phase of the flow signal extracted by the SST, (a6) synchrogram and the resulting synchrogram index (0.35) in a desynchronized patient. (b1) Electrocardiogram, (b2) IHR, (b3) phase of the IHR extracted by the SST, (b4) respiratory flow signal, (b5) phase of the flow signal extracted by the SST, (b6) synchrogram and its synchrogram index (0.89) in a synchronized patient. 
(a)

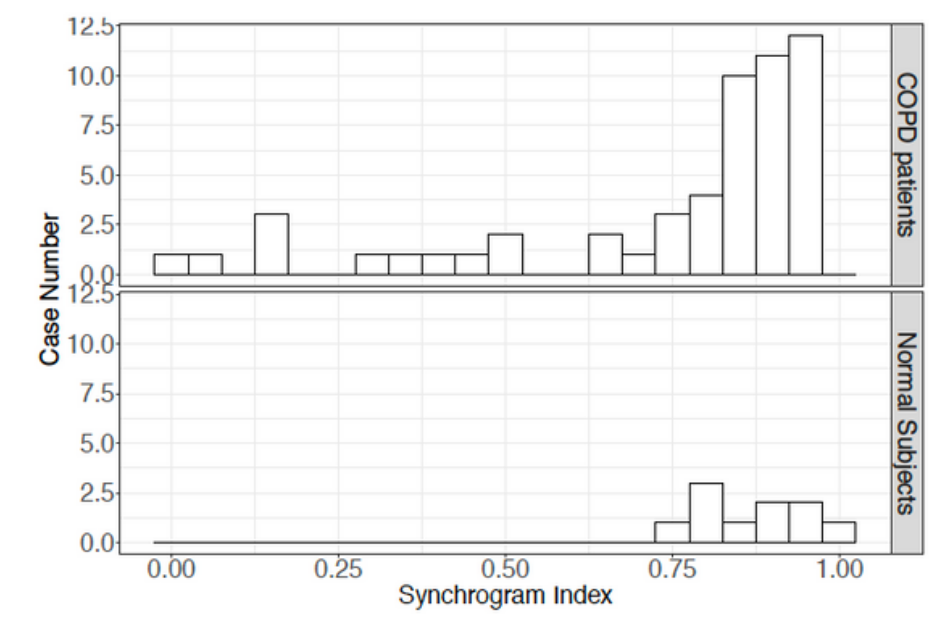

(c)

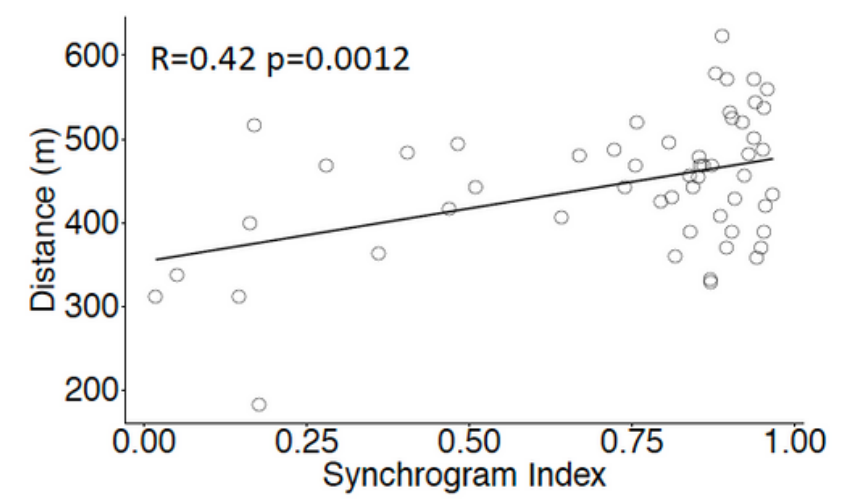

(b)

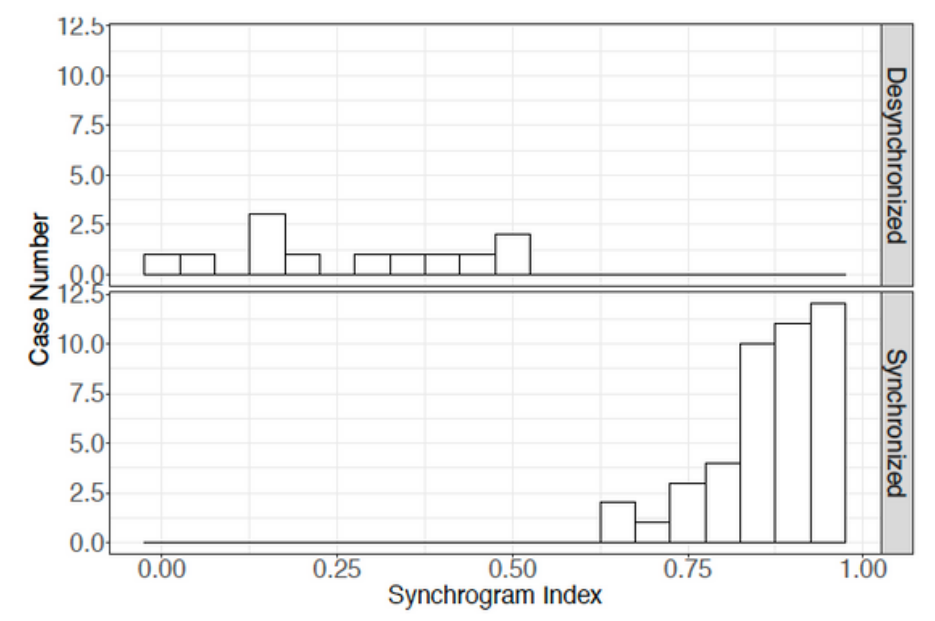

(d)

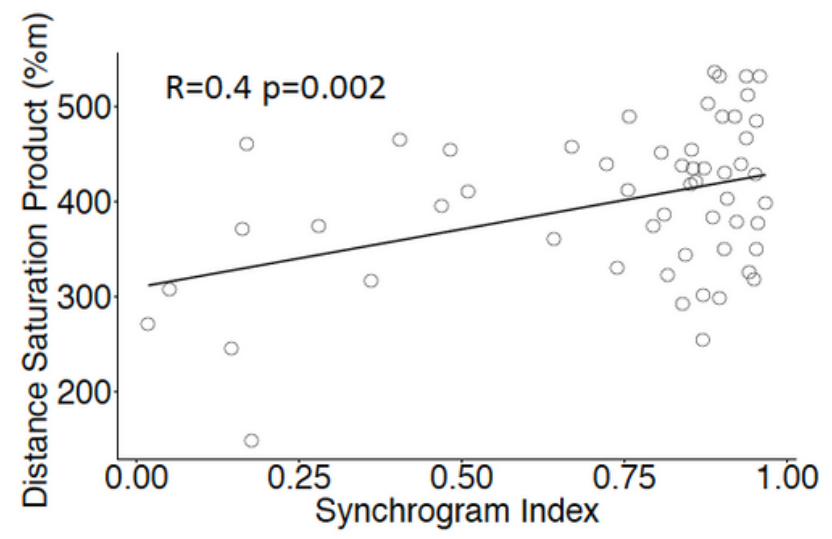

Figure 3

(a) Distribution of synchrogram index in COPD patients and normal subjects (b) Distribution of synchrogram index in synchronized group and desynchronized group. (c) Scatterplot of synchrogram index against distance $(\mathrm{m})$ from six-minute walking test of all patients $(\mathrm{d})$ Scatterplot of synchrogram index against distance saturation product $(\mathrm{m} \%)$ from six-minute walking test of all patient. 